\title{
Influence of Gravity on the Sliding Angle of Water Drops on Nanopillared Superhydrophobic Surfaces
}

\author{
Hao Li ${ }^{*, a, c}$, Tianyu Yan ${ }^{b}$, Kristen A. Fichthorn*, ${ }^{*}$ \\ a School of Materials Science and Engineering, Shandong University of Science and Technology, \\ Qingdao, 266590, China \\ ${ }^{b}$ Department of Chemical Engineering, The Pennsylvania State University, University Park, \\ Pennsylvania 16802, United States \\ ${ }^{\mathrm{c}}$ National Engineering Laboratory of Offshore Geophysical and Exploration Equipment, China \\ University of Petroleum, Shandong, 266580
}




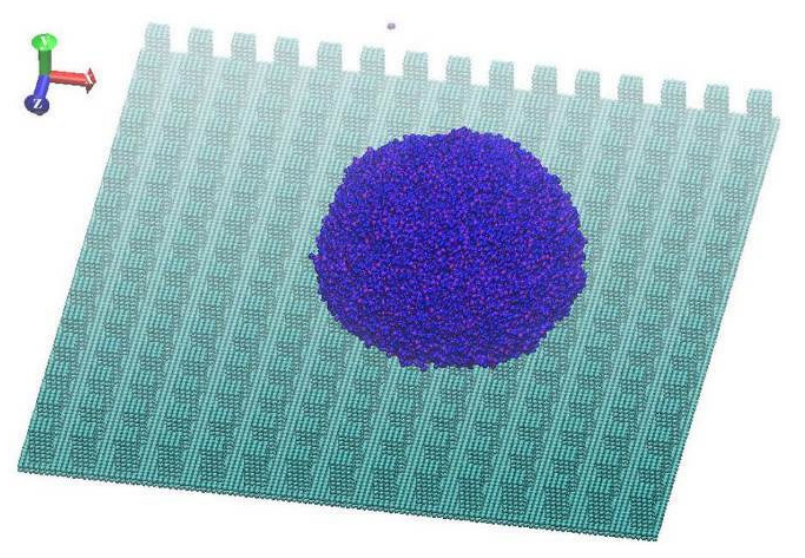

Figure S1. View of a drop on a nanopillared surface with $B o=0.15$ and $\varepsilon_{\text {surf }}=0.19 \mathrm{kcal} / \mathrm{mol}$.
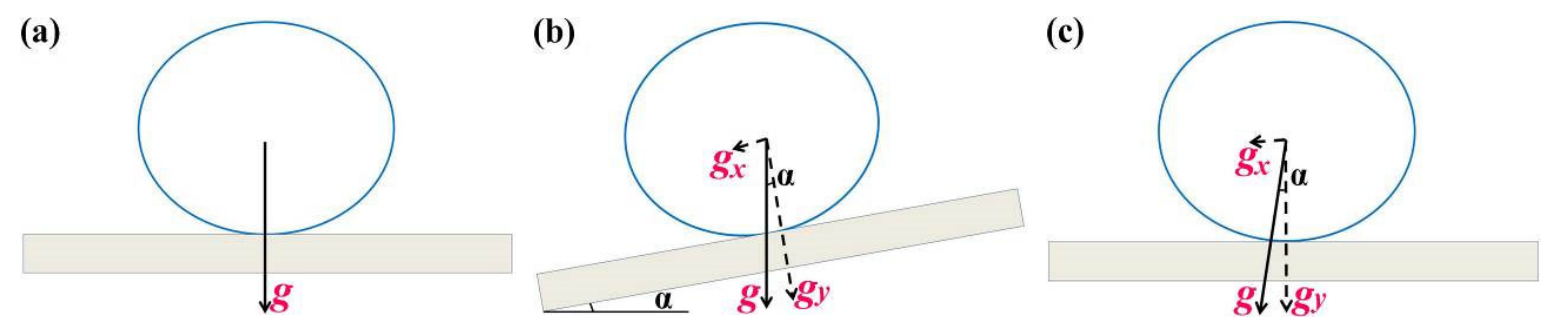

Figure S2. (a) The gravitational force $g$ for a droplet on a solid surface. (b) The $x$-and $y$-components of the gravitational force $g_{x}$ and $g_{y}$ when the surface is tilted by an angle of $\alpha$. (c) The simulated gravitational forces in MD simulations, which are implemented for a flat surface. 


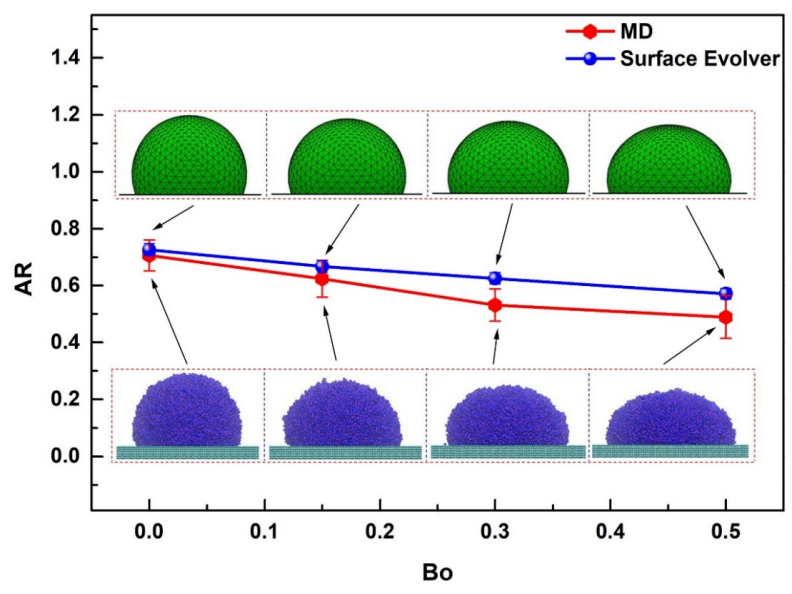

Figure S3. The aspect ratio (AR) as a function of $B o$ for MD simulations and continuum calculations using Surface Evolver. The insets show configurations of the drops with MD and Surface Evolver for $B o=0.0,0.15$, 0.30 and 0.5 .

(a)
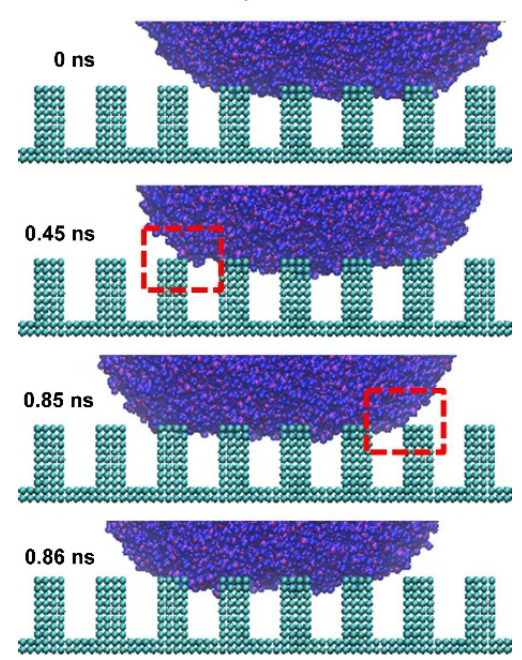

(b)
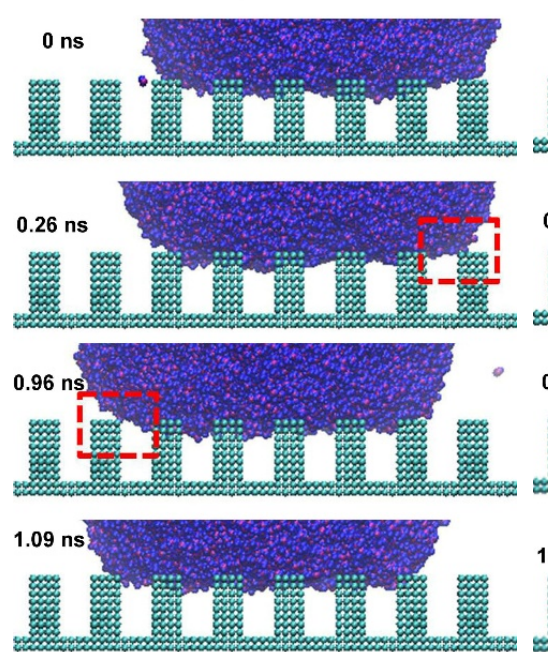

(c) Direction of Motion
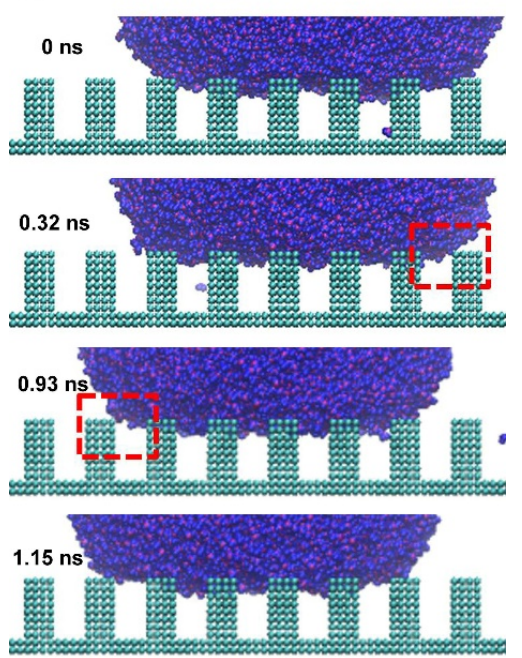

Figure S4. Side view of nanoscale water droplets and the advancing and receding contact lines moving on a nanopillared surface with different values of $B o$ : (a) 0.10 - the advancing contact line moves first and the drop stretches from covering four pillars to covering five pillars before covering four pillars in the final state; (b) 0.20 and (c) 0.25 - the receding contact line moves first and and the drop contracts from covering six pillars to covering five pillars before stretching again to cover six pillars. 

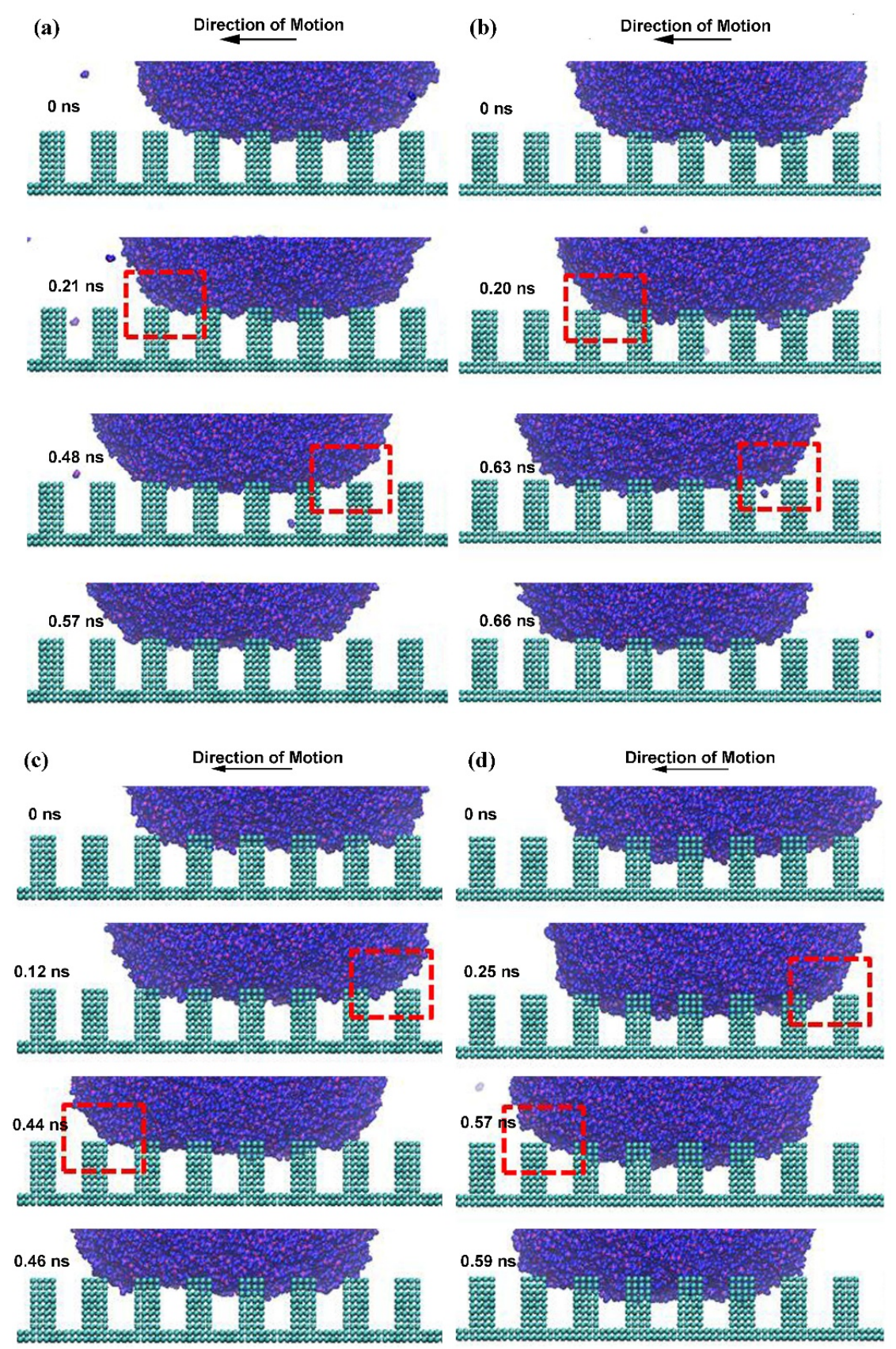

Figure S5. Side view of water droplets at the advancing and receding contact line moving on nanopillared surfaces with different LJ energy parameter $\left(\varepsilon_{\text {surf }}\right):$ (a) $0.15 \mathrm{kcal} / \mathrm{mol}$ and (b) $0.19 \mathrm{kcal} / \mathrm{mol}$ - the advancing contact line moves first and the drop stretches from covering four pillars to covering five pillars before covering four pillars in the final state; (c) $0.23 \mathrm{kcal} / \mathrm{mol}$ and (d) $0.27 \mathrm{kcal} / \mathrm{mol}$ - the receding contact line moves first and and the drop contracts from covering six pillars to covering five pillars before stretching again to cover six pillars. 

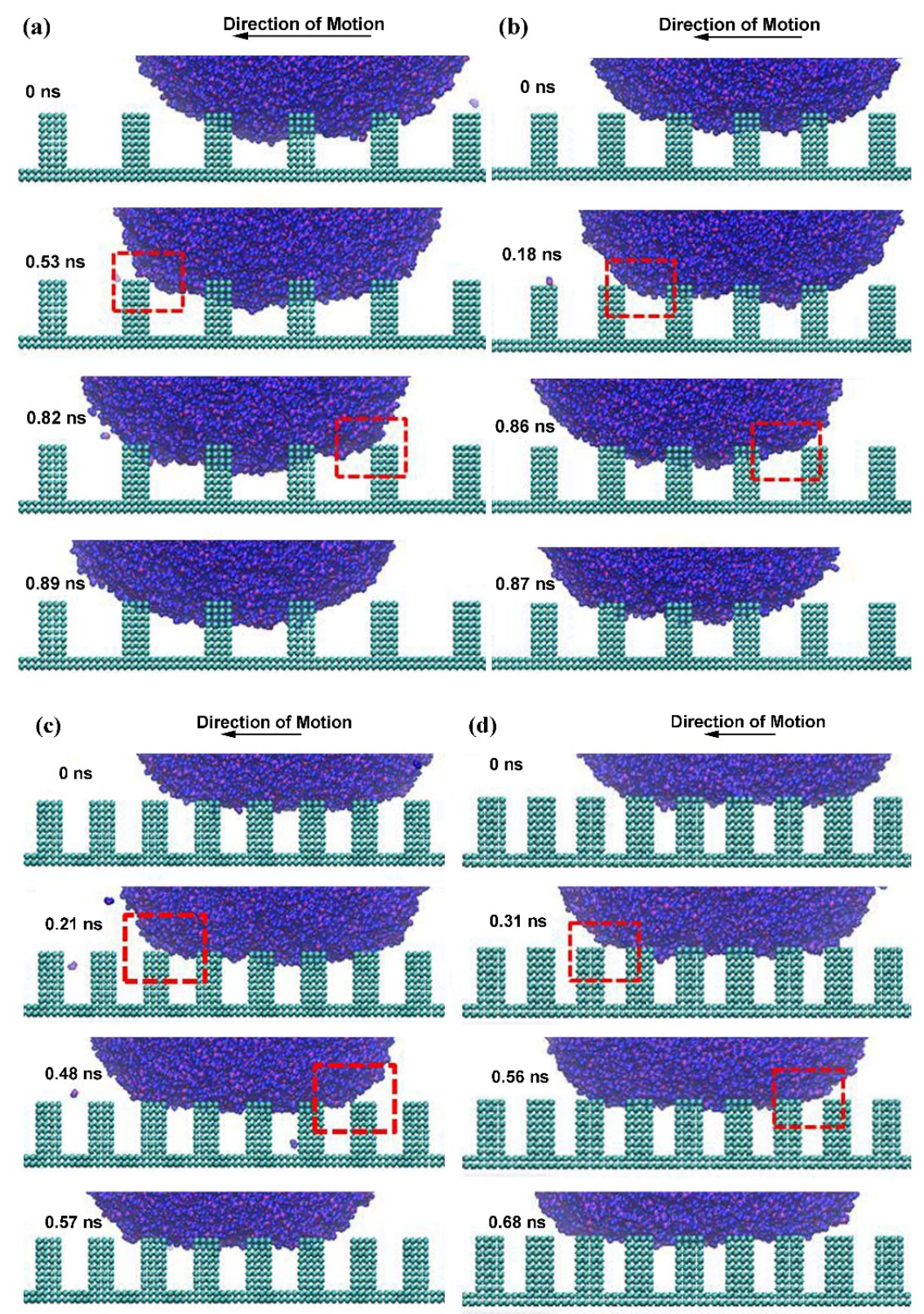

Figure S6. Side view of water droplets at the advancing and receding contact line moving on nanopillared surfaces with different fractions of water-solid interface area (f): (a) 0.11 (G8W4H8) and (b) $0.16(G 6 W 4 H 8)$ the advancing contact line moves first and the drop stretches from covering three pillars to covering four pillars before covering three pillars in the final state; (c) 0.25 (G4W4H8) - the advancing contact line moves first and the drop stretches from covering four pillars to covering five pillars before covering four pillars in the final state; (d) 0.33 (G3W4H8) - the advancing contact line moves first and the drop stretches from covering five pillars to covering six pillars before covering five pillars in the final state. 


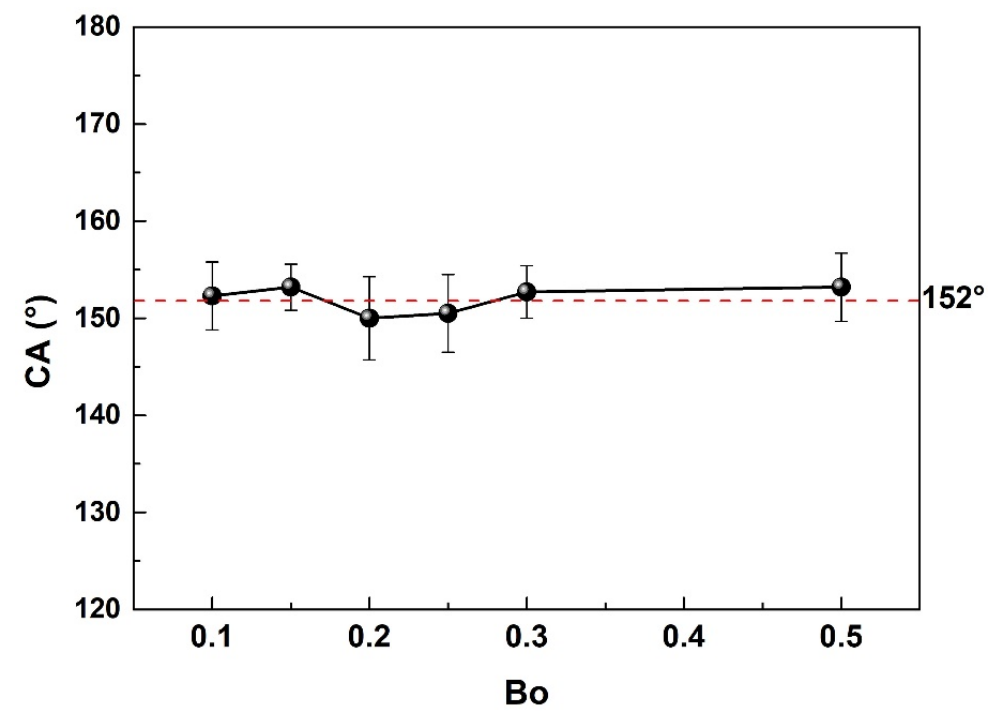

Figure S7. Static contact angle $\left(\theta_{\mathrm{s}}\right)$ of water droplets on nanopillared surfaces as a function of $B o$. The droplet includes 36,000 water molecules at $T=298.16 \mathrm{~K}, \sigma=2.858 \mathrm{~A}$, and $\varepsilon_{\text {surf }}=0.19 \mathrm{kcal} / \mathrm{mol}$.
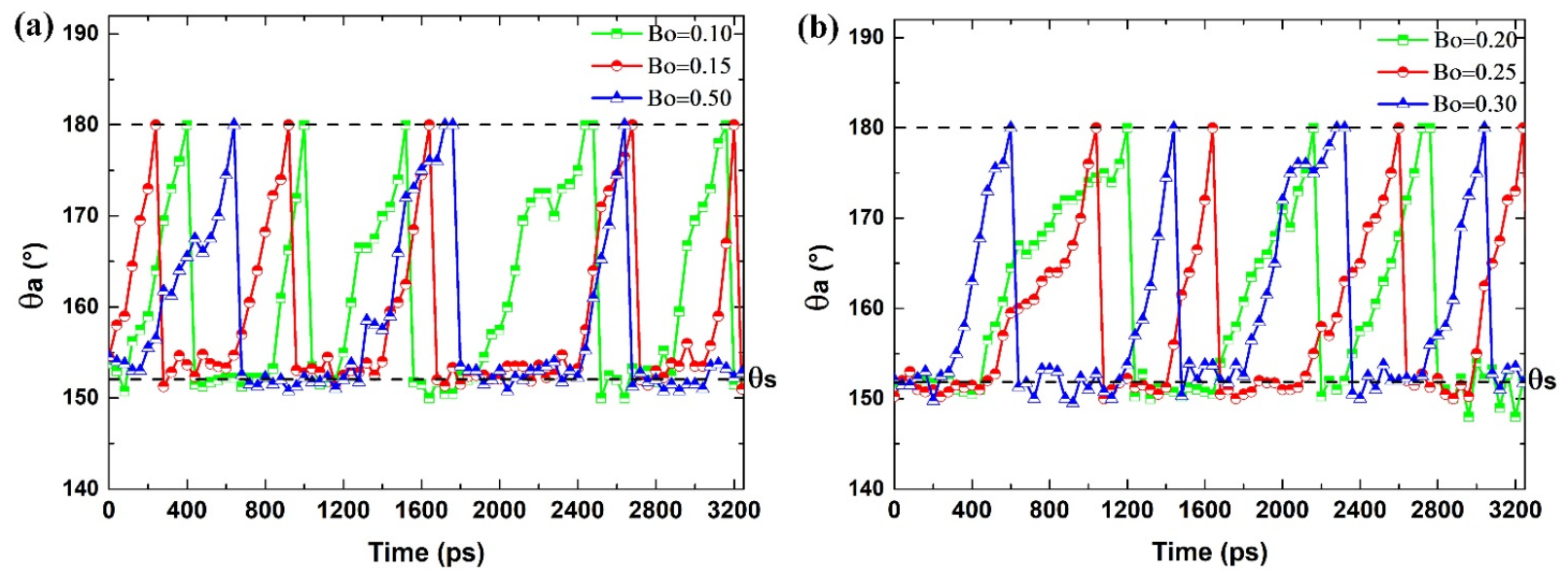

Figure S8. Dynamic advancing contact angles $\left(\theta_{\mathrm{a}}\right)$ from MD simulation trajectories plotted versus time on nanopillared surfaces with different $B o$. The contact lines are pinned on the outside (a) Bo of 0.10, 0.15, 0.50, and inside (b) Bo of 0.20, 0.25, 0.30, edge of the pillar top. 

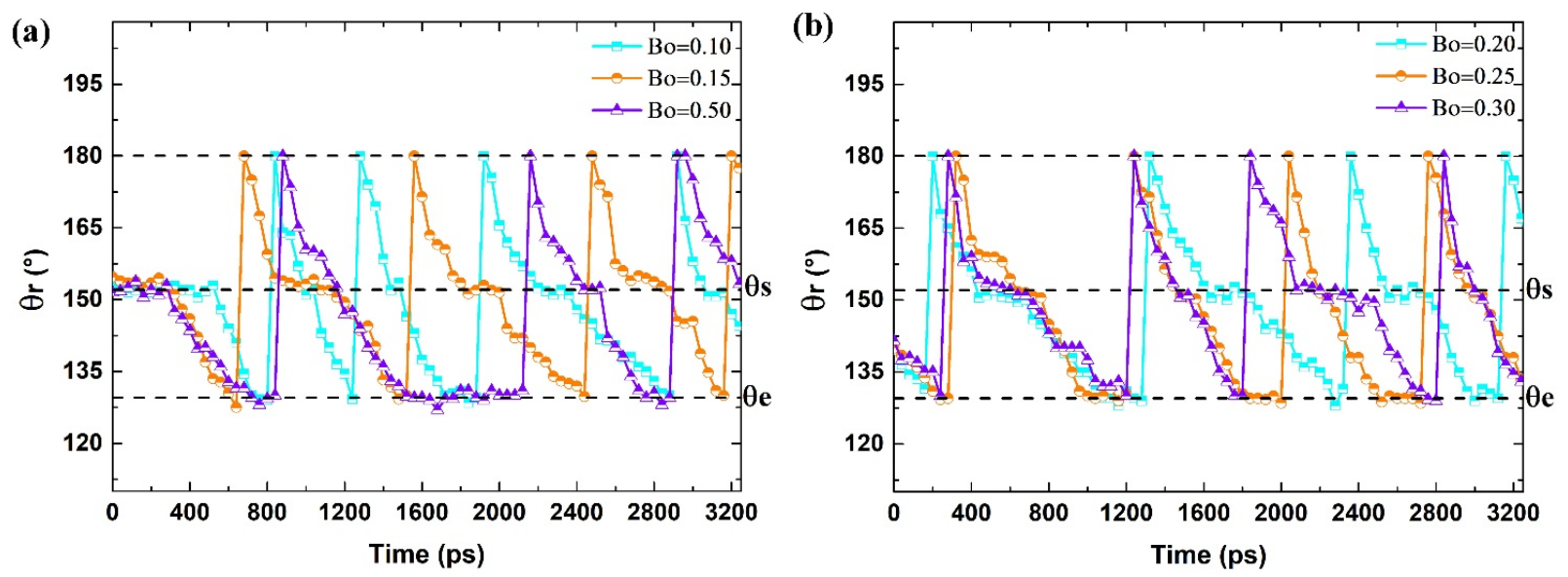

Figure S9. Dynamic receding contact angles $\left(\theta_{\mathrm{r}}\right)$ on the nanopillared surface with different Bo measured from

MD simulation trajectories plotted versus time. The contact lines are pinned on the outside (a) Bo of $0.10,0.15$,

0.50 , and inside (b) Bo of $0.20,0.25,0.30$, edge of the pillar top. 


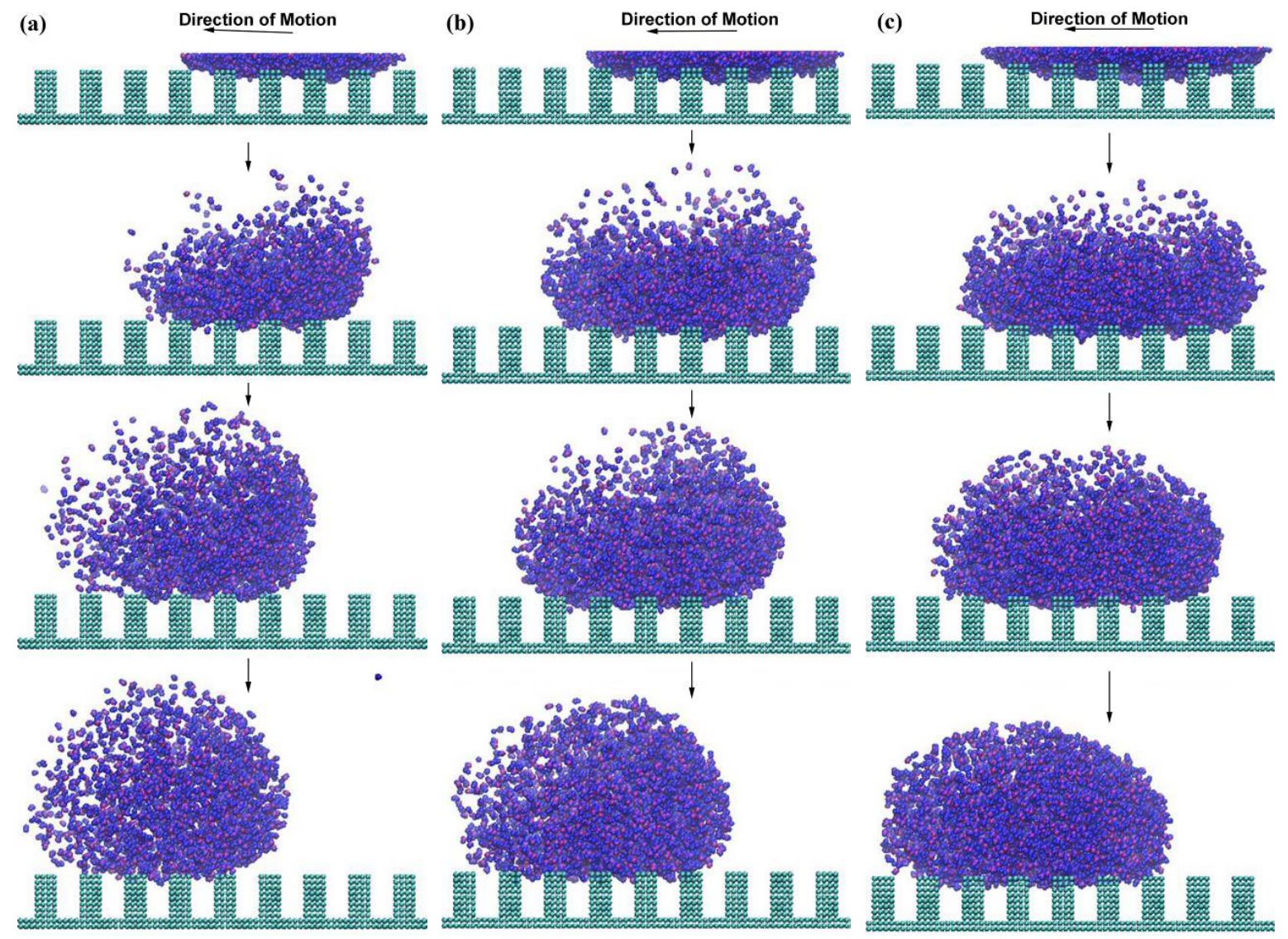

Figure S10. Side-view snapshots of water molecules initially at the liquid-solid interface at different times as the drop moves for different values of $B o:$ (a) 0.15 , (b) 0.30 , and (c) 0.50 . 


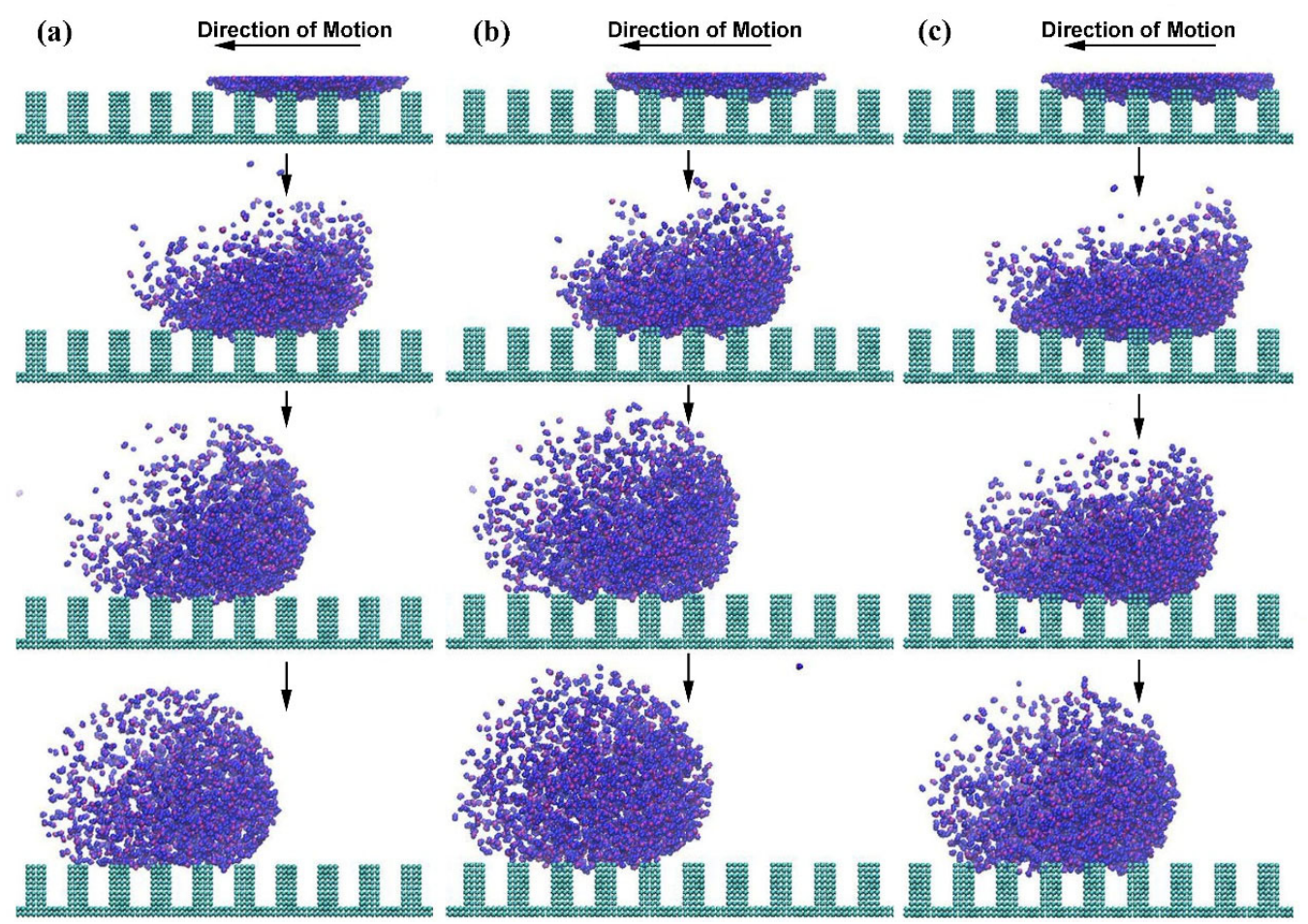

Figure S11. Side view of the motion of water molecules initially at the liquid-solid interface between a water droplet and a nanopillared surface with different LJ energy parameter ( $\varepsilon_{\text {surf }}$ ): (a) $0.15 \mathrm{kcal} / \mathrm{mol}$, (b) $0.19 \mathrm{kcal} / \mathrm{mol}$, (c) $0.23 \mathrm{kcal} / \mathrm{mol}$.

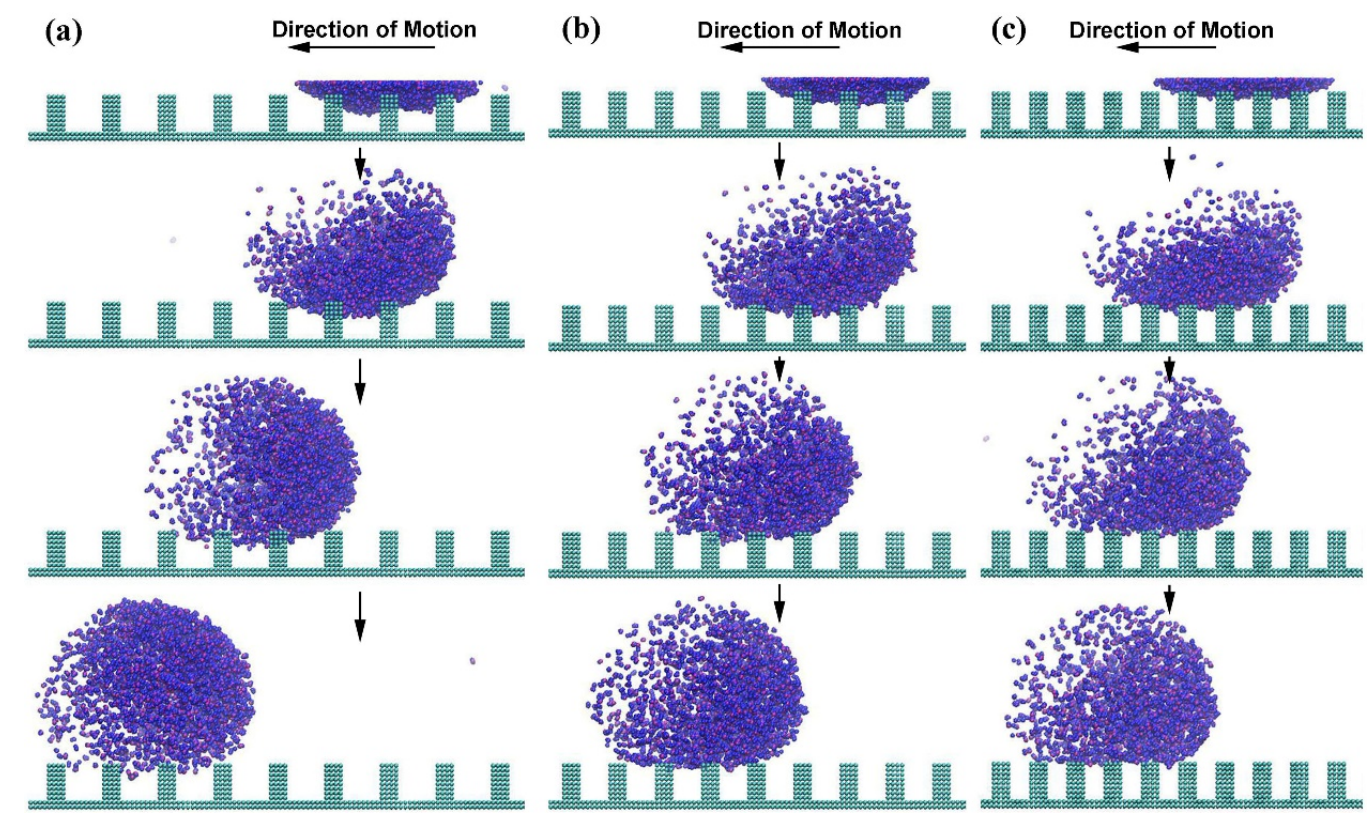

Figure S12. Side view of the motion of water molecules initially at the liquid-solid interface between a water droplet and a nanopillared surface with different fractions of water-solid interface area (f): (a) 0.11 (G8W4H8), (b) 0.16 (G6W4H8), (c) 0.25 (G4W4H8). 
(a1)

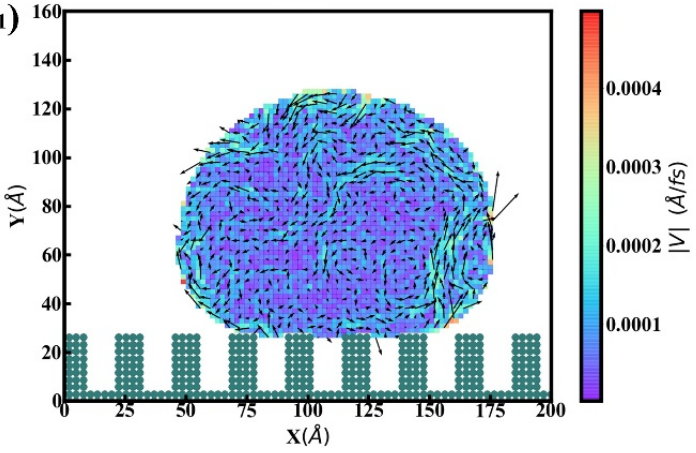

(b1)

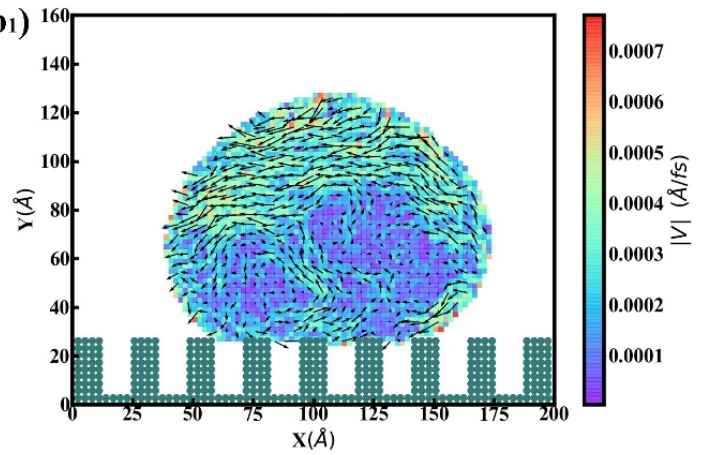

(c1)

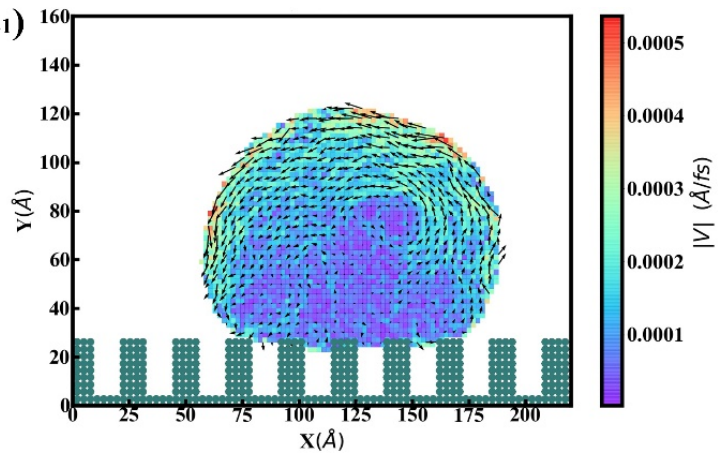

(a2)

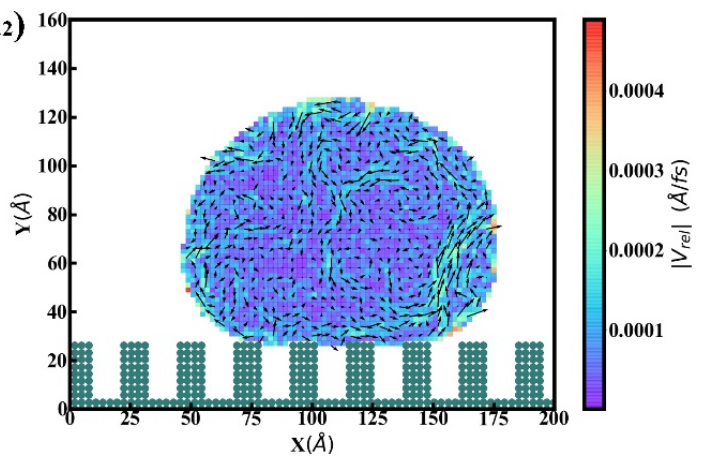

(b2)

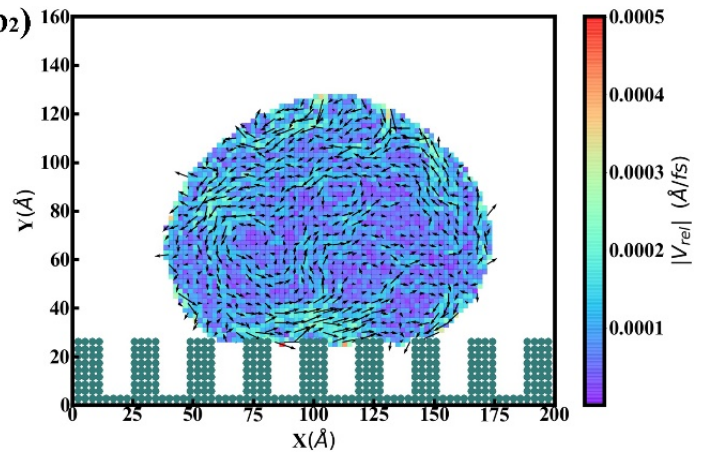

(c)

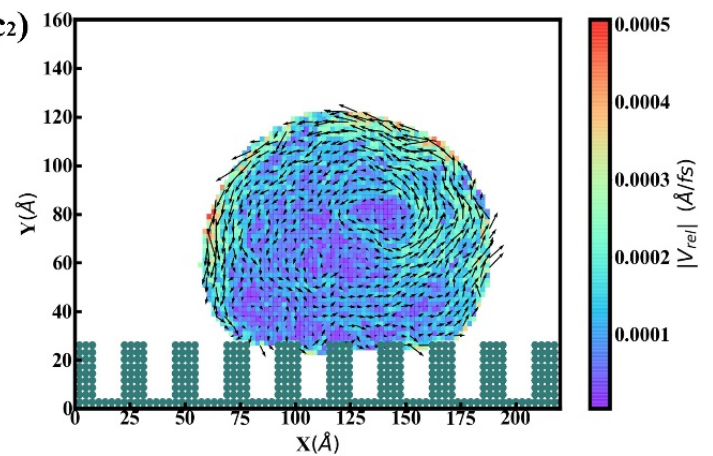

Figure S13. Velocity fields of a water droplet in the $x-y$ plane perpendicular to a nanopillared surface with different LJ energy parameters ( $\varepsilon_{\text {surf }}$ ): (a) $0.15 \mathrm{kcal} / \mathrm{mol}$, (b) $0.19 \mathrm{kcal} / \mathrm{mol}$, (c) $0.23 \mathrm{kcal} / \mathrm{mol}$. Here, (a1), (b1) and (c1) are the velocity field (v) and (a2), (b2) and (c2) are the velocity field relative to the droplet center-ofmass velocity $\left(v_{\text {rel }}\right)$. 
(a1)

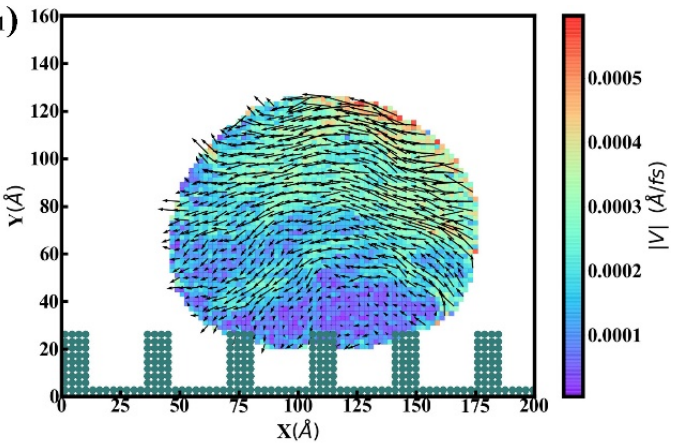

(b1)

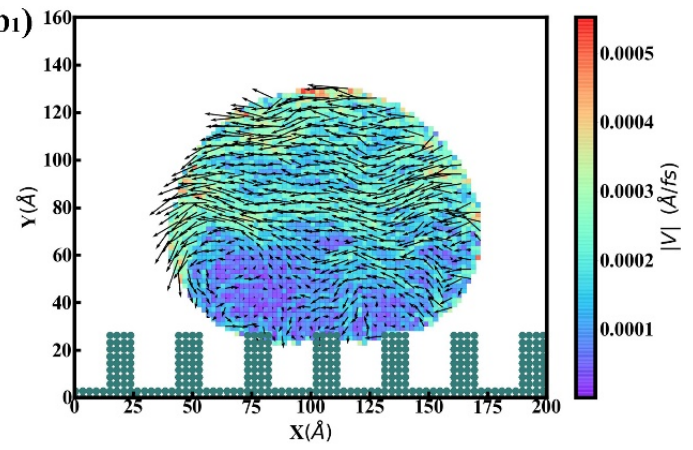

(c1)

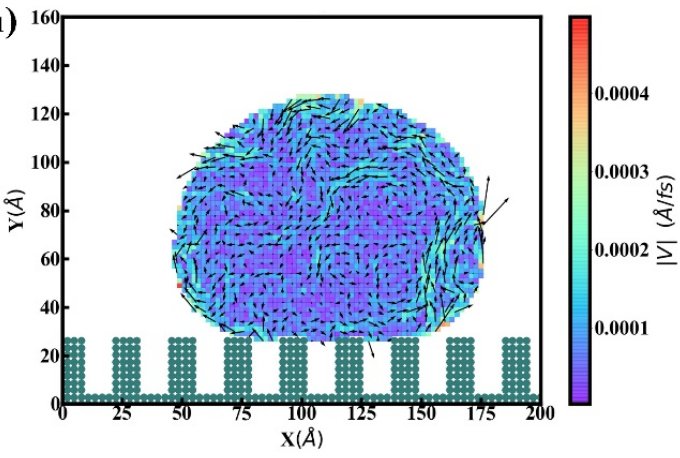

(a2)

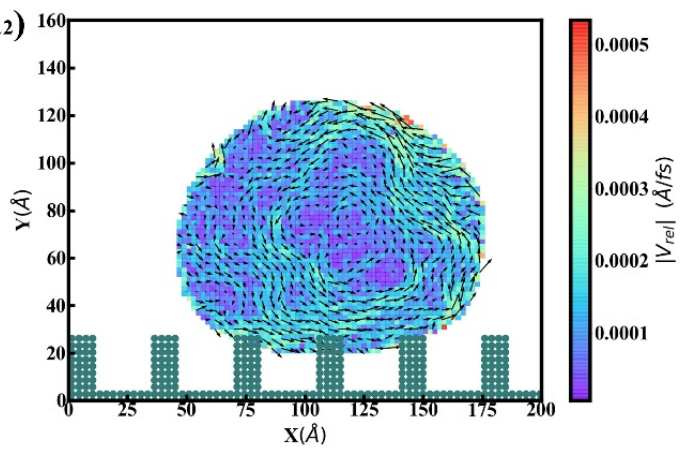

(b2)

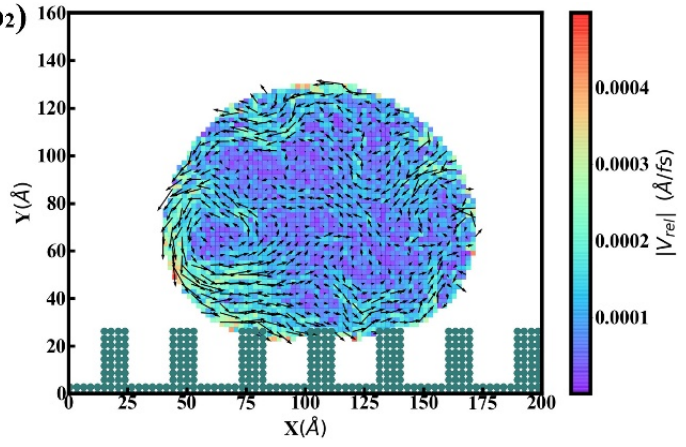

(c2)

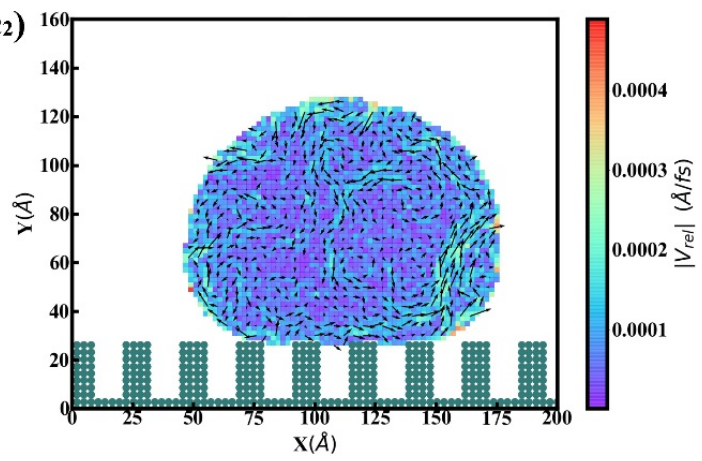

Figure S14. Velocity fields of a water droplet in the $x-y$ plane perpendicular to a nanopillared surface with different fractions of water-solid interface area (f): (a) 0.11 (G8W4H8), (b) 0.16 (G6W4H8), (c) 0.25 (G4W4H8). Here, (a1), (b1) and (c1) are the velocity field (v) and (a2), (b2) and (c2) are the velocity field relative to the droplet center-of-mass velocity $\left(v_{\text {rel }}\right)$. 